\title{
Funnel states as mediators of Born-Oppenheimer breakdown in reactions at an avoided crossing
}

\author{
Thomas C. Allison, ${ }^{a}$ Steven L. Mielke, ${ }^{a}$ David W. Schwenke ${ }^{b}$ and Donald G. Truhlar ${ }^{a *}$ \\ ${ }^{a}$ Department of Chemistry and Supercomputer Institute, University of Minnesota, Minneapolis, \\ MN 55455-0431, USA \\ ${ }^{b}$ NASA Ames Research Center, Mail Stop 230-3, Moffett Field, CA 94035-1000, USA
}

\begin{abstract}
Accurate quantum dynamics calculations are described for a series of three-body model systems exhibiting closely avoided crossings of potential energy surfaces in the vicinity of the reaction barrier. In particular, the surfaces show avoided crossings of bond-switching diabatic states in the vicinity of a saddle point. The dynamics calculations are carried out by linear algebraic variational methods with diabatic electronic basis functions. The coupling of electronically non-adiabatic effects to barrier crossings leads to qualitatively new kinds of quantum effects on the chemical reactivity. We find strong non-adiabatic effects on reaction probabilities due to funnel resonances with weaker effects (typically 2-20\%) off resonance.
\end{abstract}

London's adiabatic hypothesis ${ }^{1}$ that ordinary chemical reactions take place in a single electronic state and hence can be viewed as governed by a potential energy surface is so ingrained in everyday thinking about reactions of species in their ground electronic states that we usually ignore the fact that potential energy surfaces and force fields (which are the gradient fields of the potential energy surfaces) are only approximate concepts. The justification of the adiabatic hypothesis in terms of the Born-Oppenheimer separation of electronic and nuclear motions ${ }^{2}$ is well known. However, for reactions of electronically excited species, e.g., photochemical reactions, the branching ratios to various products often depend on the probability of hopping to the ground state surface, and considerable progress has been made by recognizing that such hops are most likely to occur in the regions where the upper and lower surfaces approach closely; such regions are called avoided crossings, avoided intersections or funnels. $^{3-7}$ (In some cases the surfaces actually intersect in a region of lower dimensionality, ${ }^{3-8}$ but in such cases electronically non-adiabatic collisions are expected to be strong not only at intersections but also in the much wider region of narrowly avoided intersection that surrounds the intersection. Hence, whether or not surfaces intersect, non-adiabatic dynamics are dominated by regions of avoided intersection.)

Salem ${ }^{9}$ classified avoided and conical intersections into six types, labelled A-E and $\mathrm{B}^{\prime}$. A type $\mathrm{B}^{\prime}$ avoided intersection occurs if a system switches between alternative bonding patterns corresponding to different valence bond descriptions, e.g., $\mathrm{A} \cdot+\mathrm{B}-\mathrm{C} \rightarrow \mathrm{A}-\mathrm{B}+\mathrm{C} \cdot$ or $\mathrm{A}-\mathrm{B}+\mathrm{C}-\mathrm{D} \rightarrow \mathrm{A}-\mathrm{C}+\mathrm{B}-\mathrm{D}$. The loss of electron exchange energy on switching bonds has long been recognized as the source of the energy barrier in atom transfer reactions, ${ }^{10}$ and a two-state valence bond treatment not only provides a semiquantitative prediction of the barrier height ${ }^{11}$ but also predicts the qualitative fact that the energy gap between the upper and lower adiabatic surfaces is smallest at the location of the barrier in the lower surface, ${ }^{12}$ i.e., the transition-state ridge on the lower surface is associated with a trough in the separation between the surfaces ${ }^{13}$ (although these geometric features need not coincide perfectly ${ }^{14}$ ).

A small gap between the ground and excited states can lead to interesting quantum effects associated with coupling of electronic and nuclear motion; such effects are critical for explaining electrical resistivity ${ }^{15}$ and outer-sphere electron transfer. ${ }^{16}$ What about ordinary chemical reactions, with their somewhat larger gaps? Butler and co-workers ${ }^{17}$ have pointed out that one might observe interesting effects of small gaps in Woodward-Hoffmann forbidden ${ }^{9,18}$ reactions, which are a classic case of bond switching reactions with high barriers and/or weakly interacting diabatic states. (Diabatic electronic states $^{19,20}$ are a general name for any states whose physical character changes slowly with geometry, such as single valence bond structures or single-configuration molecular orbital states, prior to diagonalizing the configuration interaction Hamiltonian.) In specific cases, the lowest energy path on the ground-state surface might well be a shoulder ${ }^{21,22}$ of a symmetry-allowed conical intersection, but the gap at the saddle point on the shoulder might be as small as a 'remnant' of the nearby conical intersection. Butler and co-workers ${ }^{17}$ interpreted their photochemical branching ratios in terms of significant non-reactive non-adiabatic reflection ${ }^{23}$ at regions of avoided crossing such that the height of the barrier on the lower surface is not as important as the probability of crossing it adiabatically. In addition to the system studied by Butler and co-workers, another example of a system with this kind of avoided crossing at a transition state has been provided by Palmer et al. $^{24}$

In addition to introducing a term coupling the adiabatic surfaces, a second consequence of the strong interaction of electronic states at a barrier is the contribution of a nuclearmotion term to the lower surface itself, that lowers the barrier. For systems with typical gaps at the barrier this term is small; for example, for gaps of $5-9 \mathrm{eV}$, the barrier lowering has been calculated $^{25}$ to be only $0.3-9 \mathrm{meV}\left(1 \mathrm{meV}=10^{-3} \mathrm{eV}=8.1\right.$ $\mathrm{cm}^{-1}$ ). The term will be larger for smaller gaps.

The quantum mechanical dynamical consequences of avoided crossings have been very extensively studied for onedimensional models, e.g., the well known works of Landau, ${ }^{26 a}$ Zener, $^{26 b}$ and Stückelberg ${ }^{26 c}$ and recent extensions. ${ }^{27-29}$ Multidimensional simulations have been restricted to trajectory surface hopping models, ${ }^{30}$ which are inappropriate for studying many quantum effects ${ }^{31}$ such as intermediate-state quantization $^{32}$ and tunnelling. ${ }^{33}$ Very recently it has become possible to calculate accurate quantum dynamics for threebody reactive collisions involving multiple potential energy surfaces, ${ }^{34-38}$ and in the present paper we apply accurate quantum dynamics techniques to study chemical reactions with narrow gaps at their transition states. This study provides a first attempt to use accurate multidimensional quantum dynamics calculations to explore quantum effects associated with the coupling of electronically non-adiabatic dynamics to barrier crossing. 


\section{The model}

Our model is an atom-diatom reaction, $\mathrm{A}+\mathrm{B}_{2} \rightarrow \mathrm{AB}+\mathrm{B}$, where there is a small energy gap between the ground and first excited electronic states in the region of the barrier on the lower surface. We chose to study the heavy-light combination by setting the masses of $\mathrm{A}$ and $\mathrm{B}$ equal to those of ${ }^{37} \mathrm{Cl}$ and ${ }^{1} \mathrm{H}$, respectively.

The potential energy surfaces are based on semi-empirical valence bond functional forms, ${ }^{10-12,39,40}$ which should have realistic generic shapes, but they do not represent a specific system. In particular, we created a series of five sets of two coupled potential energy surfaces defined in the diabatic representation. Each surface set is defined by the lower diabatic surface, called $H_{11}^{\mathrm{d}}$, the upper diabatic surface, $H_{22}^{\mathrm{d}}$, and their coupling, $H_{12}^{\mathrm{d}}$. Diagonalizing the $\boldsymbol{H}^{\mathrm{d}}$ matrix yields the two adiabatic surfaces, $V_{1}$ and $V_{2}$. The $\boldsymbol{H}^{\mathrm{d}}$ matrices are chosen such that the implied lower adiabatic surface, $V_{1}$, is almost exactly the same for all five cases, and it is very nearly the same as the $\mathrm{ClH}_{2} \mathrm{G} 3$ surface, ${ }^{22}$ which is the most accurate available potential energy surface for $\mathrm{ClH}_{2}$; this ensures in the first place that the lower adiabatic surface has a realistic shape, but also, more importantly for interpreting the results, it ensures that the lower adiabatic surface is the same in all five test cases. Thus we will be able to interpret the results as showing the effect of reducing the gap for a fixed ground-state surface. The classical endoergicity of the ground-state adiabatic surface is $0.131 \mathrm{eV}$ for all five cases.

The coupling matrix element $H_{12}^{\mathrm{d}}$ vanishes whenever $\mathrm{A}$ is infinitely far from B and also when either B-type atom is infinitely far from the remaining $\mathrm{AB}$ diatomic. Thus the adiabatic and diabatic surfaces become identical in these asymptotic regions. In what follows the zero of energy for diagonal elements of the $\boldsymbol{H}^{\mathrm{d}}$ and $\boldsymbol{V}$ potential matrices and for the total energy $E$ in the dynamics calculations is defined such that $H_{11}^{\mathrm{d}}=V_{1}=0$ when $\mathrm{A}$ is infinitely far from $\mathrm{B}_{2}$, with $\mathrm{B}_{2}$ at its equilibrium internuclear distance, $r_{\mathrm{e}, \mathrm{BB}}=0.741 \AA$.

The $\boldsymbol{H}^{\mathrm{d}}$ matrix elements are chosen to have physical shapes corresponding to a conical intersection of the $\mathrm{B}^{\prime}$ type discussed above. The conical intersection occurs in $C_{2 \mathrm{v}}$ geometry at the same values of the internuclear distances and same energy for all five surface sets, namely $r_{\mathrm{AB}}=3.281 a_{0}, r_{\mathrm{BB}}=$ $2.249 a_{0}$, and $H_{11}^{\mathrm{d}}=H_{22}^{\mathrm{d}}=V_{1}=V_{2}=2.794 \mathrm{eV}$ (note that $H_{12}^{\mathrm{d}}=0$ at the conical intersection). This is much higher in energy than the saddle point to reaction, which is $V_{1}^{\neq}=0.342$ $\mathrm{eV}$, and than the total collision energies $E$ considered in this paper, which are between 0.4 and $1.0 \mathrm{eV}$. Thus the important non-adiabatic interactions are not those at the conical intersection but rather the avoided intersections at lower energy. The $\boldsymbol{H}^{\mathrm{d}}$ matrices are further chosen such that the smallest gap $\Delta E$ [the gap is defined as the minimum value of the separation of the adiabatic surfaces, i.e., $\left.\Delta E \equiv \min \left(V_{2}-V_{1}\right)\right]$ occurs very close to the saddle point. The five surface sets (I-V) differ in the value of $\Delta E$, which is equal to 1.2 (I), 12 (II), 25 (III), 37 (IV), and $50(\mathrm{~V}) \mathrm{meV}$. (In spectroscopic units these gaps range from 10 to $400 \mathrm{~cm}^{-1}$.)

Full details of the functional forms of the $\boldsymbol{H}^{\mathrm{d}}$ matrices are given in the Appendix. The $\boldsymbol{H}^{\mathrm{d}}$ matrix of eqn. (A13) of the Appendix completely defines the surface set for the scattering calculations. Diagonalization of $\boldsymbol{H}^{\mathrm{d}}$ yields the final adiabatic surfaces $V_{1}$ and $V_{2}$ and adiabatic eigenvectors $c_{1}$ and $c_{2}$ for interpretation and plotting purposes, but $V_{1}$ and $V_{2}$ are not used explicitly for dynamical calculations:

$$
\left(\begin{array}{ll}
c_{11} & c_{21} \\
c_{12} & c_{22}
\end{array}\right)\left(\begin{array}{ll}
H_{11}^{\mathrm{d}} & H_{12}^{\mathrm{d}} \\
H_{21}^{\mathrm{d}} & H_{22}^{\mathrm{d}}
\end{array}\right)\left(\begin{array}{ll}
c_{11} & c_{12} \\
c_{21} & c_{22}
\end{array}\right)=\left(\begin{array}{cc}
V_{1} & 0 \\
0 & V_{2}
\end{array}\right)
$$

By design, the final $V_{1}$ is nearly the same for all surfaces and is very similar to the $\mathrm{G} 3$ potential energy surface. When $H_{11}^{\mathrm{d}}=$ $H_{22}^{\mathrm{d}}$, one finds $V_{2}-V_{1}=2\left|H_{21}^{\mathrm{d}}\right|$; the seam where this occurs may be called the avoided crossing seam.
Fig. 1 and 2 show two-dimensional contour maps of the lower and upper adiabatic surfaces for case $\mathrm{E}$ (the case in which the gap is $50 \mathrm{meV}=400 \mathrm{~cm}^{-1}$ ). Fig. 1 shows that the lower adiabatic surface has the typical shape of a simple barrier reaction. Fig. 2 shows that the upper adiabatic surface has a broad minimum in the saddle point region of the lower surface. The top panels of Fig. 3 and 4 show one-dimensional cuts through the adiabatic surfaces for case $\mathrm{E}$; the bottom panels of Fig. 3 and 4 show the fractional contributions, $c_{\mathrm{ij}}^{2}$, of the two diabatic states to the adiabatic ones along these onedimensional cuts. Fig. 3 shows that the region of narrowly avoided intersection between the two adiabatic surfaces is about $0.2 \AA$ wide. Fig. 4 shows that the lower adiabatic surface increases as the system bends, which is a visual confirmation of the fact that the saddle point on the lower surface is indeed collinear. More significantly, Fig. 4 shows that the separation between the two adiabatic surfaces also increases as the system bends. The upper adiabatic energy also increases as one leaves the saddle point in either direction along the quasisymmetric stretch coordinate (approximately given by a line connecting the origin to the saddle point in Fig. 1). Thus both the minimum of the funnel and its point of closest approach to the ground state are located essentially right over the saddle point. This confirms that we have succeeded in designing a system with a funnel over a saddle point.

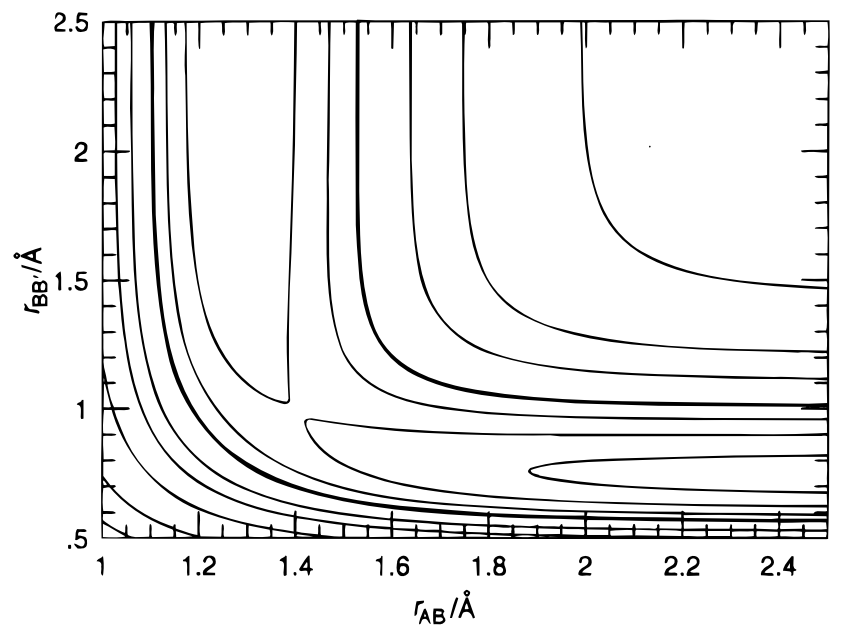

Fig. 1 Contours of the lower adiabatic surface for collinear geometries. Contours are plotted at 0.11, 0.34, 0.57, 0.80 (bold), 1.26, 1.72, $2.64,4.48$ and $6.78 \mathrm{eV}$.

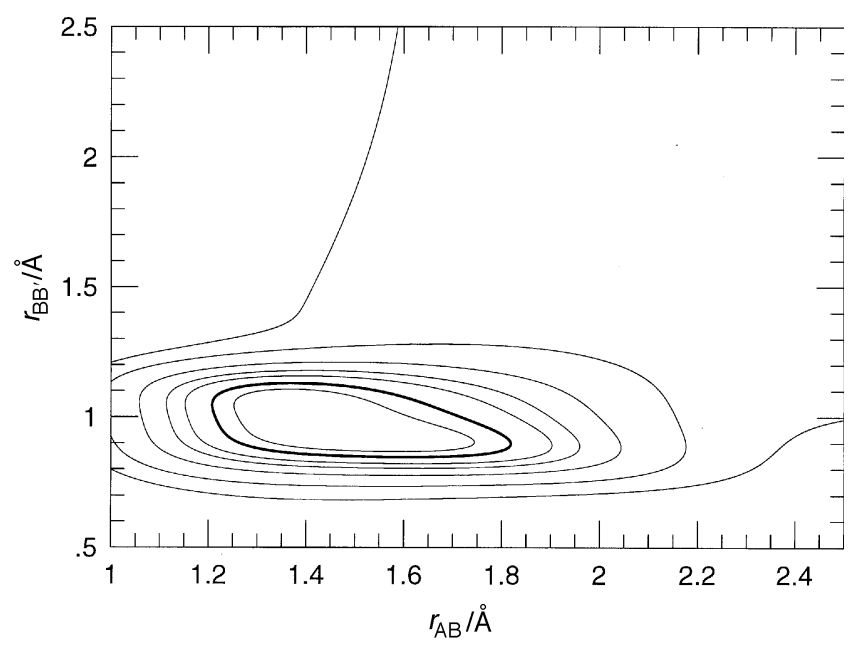

Fig. 2 Contours of the upper adiabatic surface for collinear geometries for case V (in which the gap is $400 \mathrm{~cm}^{-1}$ ). The contours plotted are the same as in Fig. 1. 


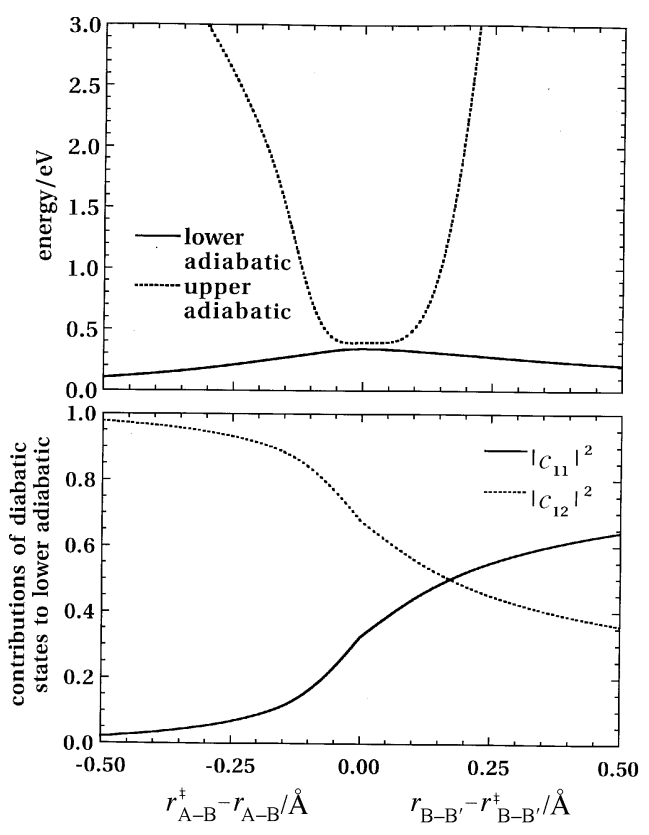

Fig. 3 Energies of and diabatic contributions to the lower and upper adiabats of the potential along the collinear minimum energy path in case $\mathrm{V}$. The abscissa labels a reaction coordinate which is negative as A approaches $\mathrm{BB}^{\prime}$; zero at the saddle point, and positive as B moves away from $\mathrm{AB}^{\prime}$.

Although Fig. 1-4 are specifically for case V, plots of the same quantities for cases I-IV look very similar, by design. The only difference is that the funnel approaches the lower surface more closely. This is illustrated by comparing cases I and $\mathrm{V}$ in Fig. 5; cases II-IV are intermediate.

\section{Computations}

All quantum dynamics calculations were carried out by linear algebraic variational scattering methods with electronically diabatic basis functions on Cray supercomputers. We used both the generalized Newton variational principle ${ }^{41-43}$

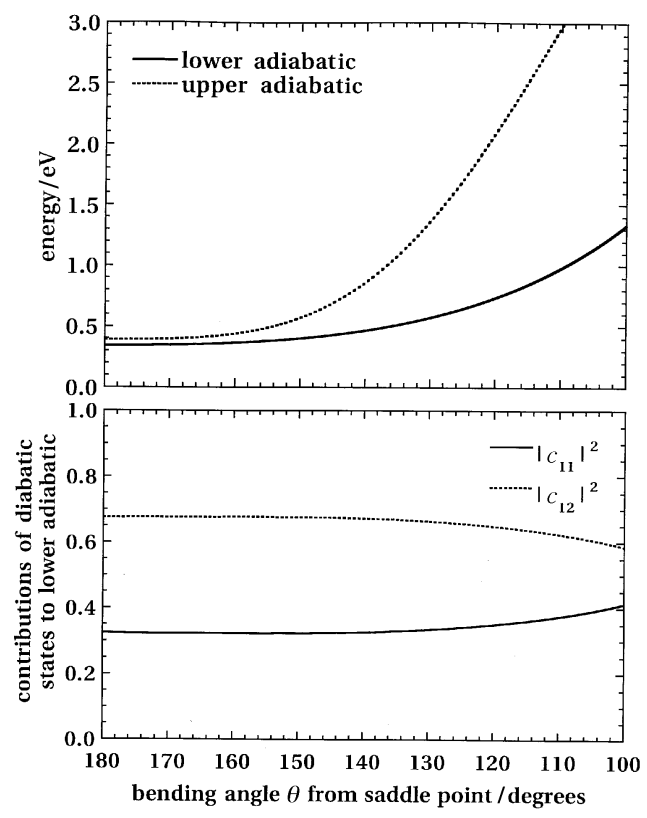

Fig. 4 Energies of the diabatic contributions to the lower and upper adiabats of case $\mathrm{E}$ as a function of the angle of bending away from the saddle point. The nearest-neighbour distances are fixed at constant values as the system is bent.

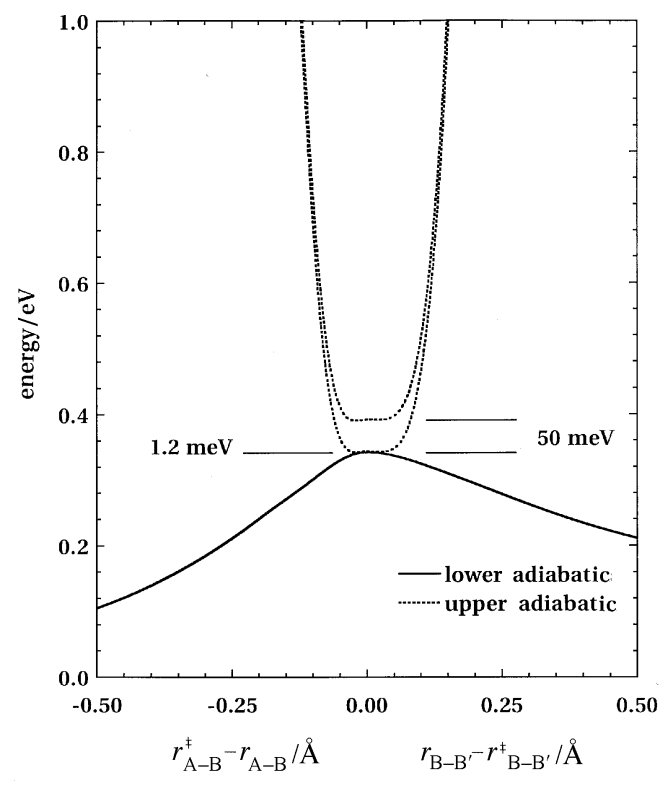

Fig. 5 Energies of the lower and upper adiabats of the potential along the collinear minimum energy path for cases I and V (in which the gaps are 10 and $400 \mathrm{~cm}^{-1}$, respectively). The abscissa is labelled as in Fig. 3.

(GNVP) and the outgoing wave variational principle ${ }^{44-47}$ (OWVP). The GNVP calculations are formulated as OWVP calculations in which the basis functions in the scattering coordinate (the radial relative translational coordinate) consist of a set of half-integrated Green's functions (HIGFs) generated from a set of evenly spaced radial Gaussian functions as explained previously. ${ }^{43,47}$ In the OWVP calculations for the present paper, we used these HIGFs as basis functions in open channels, and we used the radial Gaussian functions themselves as the basis functions in closed channels. Full details of the formulation for electronically inelastic reactive scattering, including the form of basis functions in all coordinates and the numerical procedures, are presented in full in previous publications. ${ }^{48,49}$

There are three computational steps in the linear albegraic variational calculations.

1. Compute the incoming coupled-channel distorted-wave and the coupled-channel half-integrated radial distorted-wave Green's functions directly on quadrature grids by high-order finite differences (a 13-point formula in the main part of the grid, reduced to an 8-point formula near the edges).

2. Carry out a six-dimensional integration over the coupling part of the interaction potential, the numerical solutions found in step 1, and (in the OWVP calculations) over the other basis functions. Three dimensions are integrated analytically in a molecule-fixed coordinate system, and the other three are integrated numerically.

3. Solve a linear system of equations for coefficients of the basis functions, and use the resulting solution to compute the scattering matrix (matrix of scattering amplitudes) by matrix multiplication.

For the present calculations the distortion potential coupled all vibrational quantum numbers $v$ for a given rotational quantum number $j$ and arrangement $\alpha$. We used the same basis set on each diabatic surface, with vibrational functions based on the lower adiabatic surface. Parameters characterizing the basis sets and numerical integrations for the present calculations are given as supplementary information. $\dagger$ The linear equation solution of step 3 was accomplished non-

$\dagger$ Available as supplementary data (SUPPL. No. 57199, 8 pp.) from the British Library. For details contact the Editorial Office. 
iteratively by decomposition and back substitution. All calculations were converged to plotting accuracy with respect to simultaneous variation of a sufficient sub-set of parameters to demonstrate convergence with respect to both basis sets and numerical integrations.

\section{Results and Discussion}

In all our calculations we studied the probability of reaction for zero total angular momentum as a function of energy. These probabilities represent converged quantum dynamics for the assumed diabatic surface sets and hence for the implicit coupled adiabatic representation as well. We also carried out converged dynamical calculations in the adiabatic representation in which we included only the ground adiabatic surface. Comparison of these adiabatic results to the coupled-surface results provides a measure of the effect of coupling to the funnel.

The particular version of the reaction probability on which we shall focus attention is the zero-total-angular-momentum, even-symmetry cumulative reaction probability (CRP) defined by

$$
N^{0+}(E)=\sum_{n} \sum_{n^{\prime}} P_{1 n 2 n^{\prime}}^{0+}(E)
$$

where $P_{\alpha n \alpha^{\prime} n^{\prime}}^{J S}(E)$ is the reaction probability from arrangement $\alpha$ and channel $n$ to arrangement $\alpha^{\prime}$ and channel $n^{\prime}$ at total angular momentum $J$ and with permutation symmetry $S$. As indicated in eqn. (2), we include only $J=0$ and even permutational symmetry $(+)$, where the latter corresponds to even rotational states of $B_{2}$. For this choice, we can take even and odd linear combinations of the $\mathrm{AB}+\mathrm{B}^{\prime}$ and $\mathrm{AB}^{\prime}+\mathrm{B}$ states, and reaction occurs only into the even arrangement, which is denoted by setting $\alpha^{\prime}=2$ on $P_{\alpha n \alpha^{\prime} n^{\prime}}^{0+}$, in eqn. (2). The reactant arrangement, $\mathrm{A}+\mathrm{B}_{2}$, is denoted $\alpha=1$. The sums over $n$ and $n^{\prime}$ in eqn. (2) are over all open (i.e., energetically accessible) channels of the $\alpha=1$ and $\alpha^{\prime}=2$ arrangements, respectively. Thus in simple language, $\mathrm{N}^{0+}(E)$ is the sum of all state-tostate probabilities for $\mathrm{A}+\mathrm{B}_{2} \rightarrow \mathrm{AB}+\mathrm{B}$ at zero total angular momentum considering only even rotational states of $\mathrm{B}_{2}$. The CRP is an important theoretical quantity because of the relation $^{50}$

$$
k(T)=\frac{1}{h \Phi^{\mathrm{R}}(T)} \sum_{S} \sum_{J}(2 J+1) \int d E \exp \left[-E / k_{\mathrm{B}} T\right] N^{J S}(E)
$$

where $k(T)$ is the ordinary reaction rate constant at temperature $T, h$ is Planck's constant, $\Phi^{\mathrm{R}}(T)$ is the partition function per unit volume for the reactants, and $k_{\mathrm{B}}$ is Boltzmann's constant. Thus $N^{J S}(E)$ is the total contribution of a microcanonical ensemble with given values of $E, J$, and $S$ to the thermal rate constant. We will obtain a representative sample of the dynamics by studying the $J S=0+$ ensembles as a function of $E$.

In its general features, our system was designed to have gaps similar to those in the reactions studied experimentally by Butler and co-workers. ${ }^{17}$ In the dissociation of $\mathrm{BrCH}_{2} \mathrm{COCl}$, they studied competing pathways with barriers of about 0.7 and $1.1 \mathrm{eV}$ and gaps of 2 and $50 \mathrm{meV}$. Our $\mathrm{AB}_{2}$ model system has a classical barrier height of $0.34 \mathrm{eV}$ and gaps of $1.2-50 \mathrm{meV}$. Adding zero-point energy at the saddle point raises the total energy there to $0.50 \mathrm{eV}$, which would be a zero-order estimate of the expected threshold energy for a single-surface reactive process.

The dotted curve in Fig. 6 shows our results for the adiabatic case. Interpretation of the CRP in terms of quantized transition states and a parabolic effective barrier shape along the reaction coordinate would indicate that the CRP should attain a value of one half at the energy of the lowest quantized

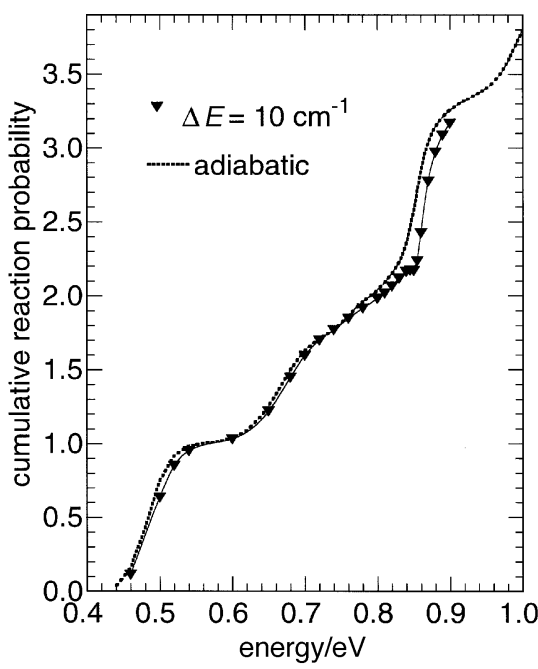

Fig. $6 \mathrm{CRP}$ as a function of total energy $E$ for the adiabatic system and for a gap of $10 \mathrm{~cm}^{-1} . J S=0+$.

level of the transition state. ${ }^{51}$ The adiabatic CRP in Fig. 6 attains a value of one half at a total energy slightly above 0.48 $\mathrm{eV}$ in reasonable agreement with the zero-order expectation in the previous paragraph.

Fig. 6 also shows the CRP for the first coupled surface case, case I. In order to keep as close as possible to the language used by the experimentalists, we will label the cases in the paper by the energy gaps in wavenumbers: I, $10 \mathrm{~cm}^{-1}$; II, 100 $\mathrm{cm}^{-1}$; III, $200 \mathrm{~cm}^{-1} ; \mathrm{IV}, 300 \mathrm{~cm}^{-1} ; \mathrm{V}, 400 \mathrm{~cm}^{-1}$. For case I $\left(10 \mathrm{~cm}^{-1}\right)$ shown in Fig. 6, the most significant deviation of the coupled-surface CRP from the adiabatic one occurs in a region from $c a$. 0.84 to $0.87 \mathrm{eV}$. Fig. 7 and 8 shows the CRPs for all five coupled-surface cases, compared with the adiabatic $\mathrm{CRP}$, in the interesting energy region above $0.8 \mathrm{eV}$. We see that as the gap increases, the energy at which the maximum deviation occurs also increases. Fig. 9 brings this out more clearly by showing the ratios of the coupled-surface CRPs to the adiabatic one, with all five cases on the same plot. Clearly the dependence of the CRP on the energy gap is quite systematic. In fact this figure strongly suggests that the deviations should be interpreted as scattering resonances. Scattering resonances are due to metastable states of the collision system, and (depending on the interference between direct processes

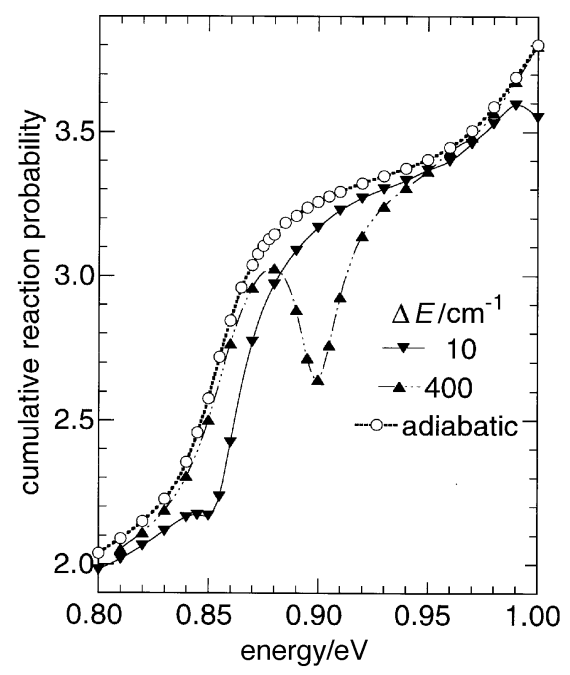

Fig. 7 Same as Fig. 6 except for a more restricted energy range, and the results are compared with the CRP for a gap of $400 \mathrm{~cm}^{-1}$. The adiabatic CRP and the CRP for a gap of $10 \mathrm{~cm}^{-1}$ are also shown. $J S=0+$. 


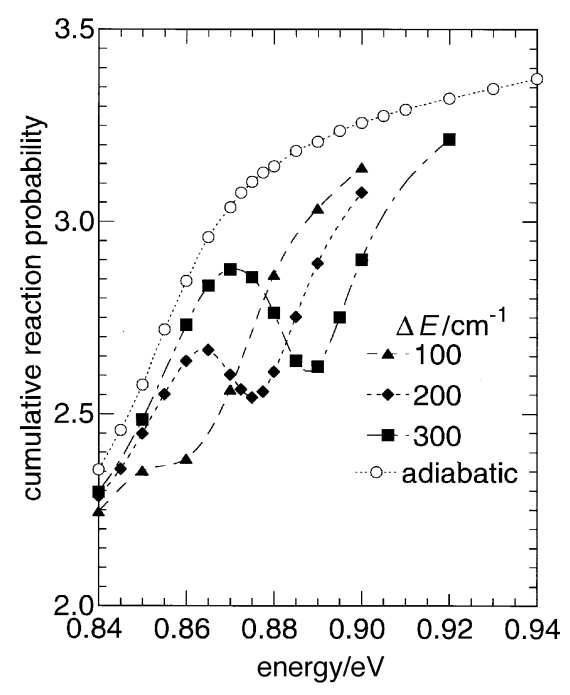

Fig. $8 \mathrm{CRP}$ as a function of total energy $E$ for the adiabatic system, and for the systems with a $100 \mathrm{~cm}^{-1}$ gap, a $200 \mathrm{~cm}^{-1}$ gap, and a 300 $\mathrm{cm}^{-1}$ gap. $J S=0+$.

and the processes that pass through the metastable states) they can lead to transition probabilities that have maxima or minima at the resonance energy, or a peak on one side with a dip on the other. ${ }^{52}$ All the physical systems considered here are seen to lead to a minimum at the resonance energy. This feature disappears if the surface coupling is turned off; hence we can associate it with metastable states associated with coupling to the upper surface. We note that in purely onedimensional $\operatorname{cases}^{28}$ with potentials like Fig. 3 or 5, complete reflections can occur at resonance, but the present multidimensional systems show only partial reflections.

Before analysing the resonances, we note that, as expected from one-dimensional models, ${ }^{28}$ the reaction (transmission) coefficients are usually smaller than the adiabatic transmission probabilities off resonance as well as on resonance. We have not analysed this quantitatively to separate the tails of the resonances from the background effects. Clearly the resonances are responsible for the dominant non-adiabatic effect.

The standard way to analyse resonances is to identify the resonance effect as the difference between the transition probability in the presence of the resonance and that in the absence of the resonance. Fig. 10 shows the differences of the resonant CRPs from the non-resonant adiabatic ones. In each

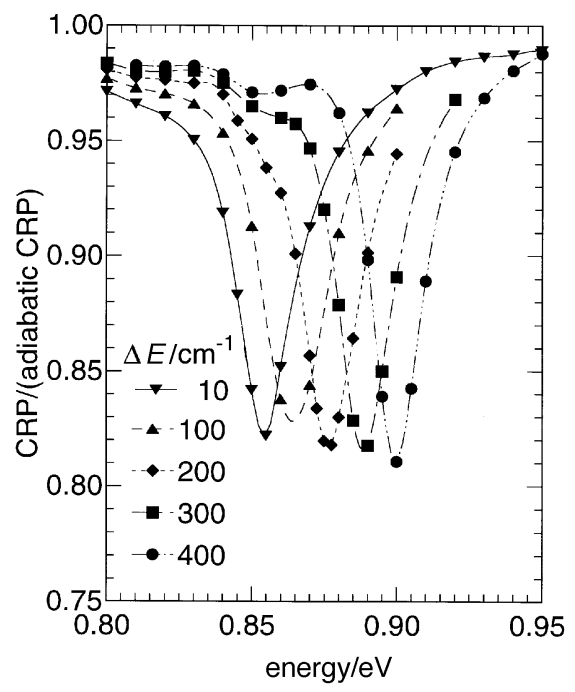

Fig. 9 Ratio of coupled-surface CRP to adiabatic one as a function of total energy $E$ for various gap sizes. $J S=0+$.

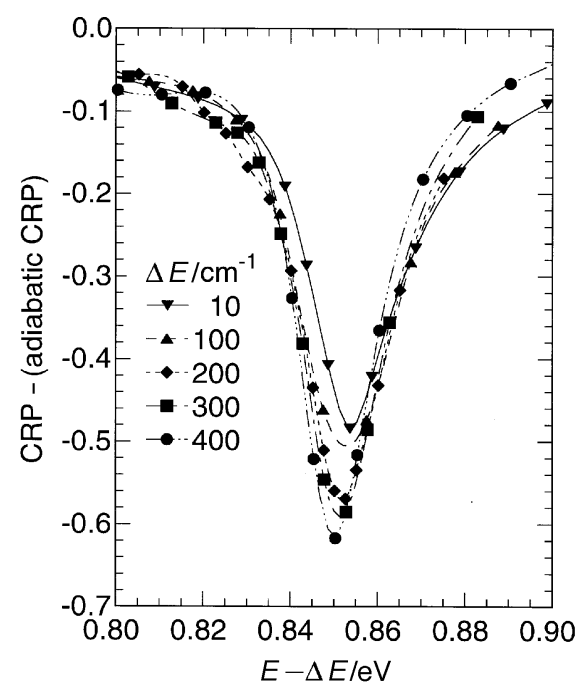

Fig. 10 Difference of coupled-surface CRP from adiabatic one as a function of $E-\Delta E$, where $E$ is the total energy, and $\Delta E$ is the gap. $J S=0+$.

case the difference is plotted as a function of $E-\Delta E$, where $\Delta E$ is the gap. This brings all five resonances into almost quantitative overlap. Since the ground adiabatic surface is essentially the same in all five cases, this indicates that in each case the resonance occurs at approximately the same energy relative to the minimum of the upper adiabatic surface. We identify this approximately constant shift between the resonance energy and the bottom of the funnel as the zero-point energy of the metastable state in the funnel. It is easily calculated from Fig. 10 and the minimum energies of the upper adiabatic surfaces (Fig. 2-5) that this zero-point energy is $c a$. $510 \mathrm{meV}$ or $4100 \mathrm{~cm}^{-1}$. Although the funnel is very anharmonic, we estimate that this zero-point energy could be rationalized by effective frequencies of $1800 \mathrm{~cm}^{-1}$ for the symmetric stretch, $2000 \mathrm{~cm}^{-1}$ for the bend, and $4400 \mathrm{~cm}^{-1}$ for the asymmetric stretch.

Another interesting observation from Fig. 10 is that the widths of the various resonances are nearly the same. In particular the full width at half maximum of the resonance feature, which is usually denoted $\Gamma$ and simply called the width, ${ }^{53}$ varies from 24 to $32 \mathrm{meV}$. From this we can calculate resonance lifetime $\Delta t$ using $^{53}$

$$
\Delta t=\hbar / \Gamma
$$

where $\hbar$ is Planck's constant divided by $2 \pi$. This yields lifetimes in the range $21-27$ fs. This can be compared with the direct transit time of the saddle-point region, which can be computed $^{51,54}$ from the widths of the 'steps' in Fig. 6; such an analysis yields 9 and 14 fs for the first few quantized transition states of the direct reaction. ${ }^{55}$

A final observation on Fig. 10 concerns the absolute magnitude of the dip in the CRP due to each resonance feature. Like the widths, this too is nearly constant across the five cases, varying from 0.49 to 0.61 . These magnitudes of the dips are not only similar to each other but also remarkably close to 0.5 , a value that leads to a very simple interpretation. To understand this interpretation we need to note that for adiabatic reaction on the potential energy surface with the mass combination studied here, variational transition state theory with multidimensional tunnelling contributions is accurate to within $14 \%$ over the entire $200-1000 \mathrm{~K}$ temperature range. ${ }^{55,56}$ Thus a picture of direct reaction occurring with a near-unity transmission coefficient is a good model, and the paradigm of quantized transition-state control of global reactivity ${ }^{54}$ explains the general increase of the CRP as a manifestation of an increasing number of accessible transition 
state levels on the lower surface. At a resonance energy though, the system becomes temporarily trapped in a metastable level of the funnel. Such a system 'forgets' whence it entered the metastable state and decays with a probability of 0.5 to reactants and of 0.5 to products. Since this is 0.5 less than the reactive-decay probability of 1.0 of the direct transition state levels accessible at this energy, the CRP dips by 0.5. (Since the metastable states have such short lifetimes, trapped systems might have a slightly larger probability of one reflection along the reaction coordinate than of two, and this could even account for the dip slightly exceeding 0.5 , but we do not place such great faith in the simple model as to expect it to reproduce the accurate scattering calculations perfectly.)

Fig. 11 partitions the CRP for the case with a $400 \mathrm{~cm}^{-1}$ gap into two components. The sum over $n$ in eqn. (2) is, in more detail, a sum over initial vibrational quantum number $v$ and initial rotational quantum number $j$. (For $J=0$ there is a unique value of the relative translational orbital angular momentum quantum number $l$ for each value of $j$.) Over the energy range considered in this paper only the $v=0$ and 1 states are open. Therefore we write

$$
N^{0+}(E)=\sum_{v=0.1} N^{0+}(v, E)
$$

where

$$
N^{0+}(v, E)=\sum_{j} \sum_{v^{\prime}} \sum_{j^{\prime}} P_{1 v j 2 v^{\prime} j^{\prime}}^{0+}(E)
$$

Fig. 11 shows that the resonance dip is approximately evenly distributed over the two components of the CRP. We conclude that both vibrationally unexcited and vibrationally

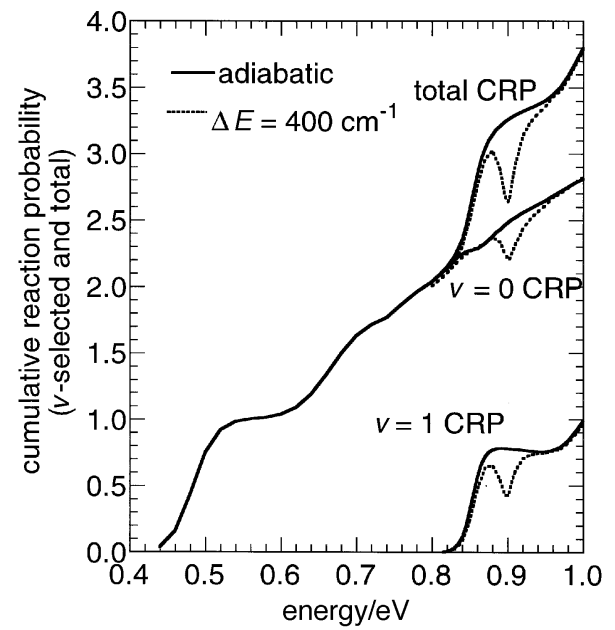

Fig. 11 Vibrational-state-selected and total CRPs as functions of total energy $E$ for the adiabatic system and for the one with a 400 $\mathrm{cm}^{-1}$ gap. $\mathrm{JS}=0+$. excited reactants can access the metastable state in the funnel. Looking at Fig. 11 from another point of view we note that the dip in the total CRP is $19 \%$, whereas the dip in the stateselected $v=1 \mathrm{CRP}$ is fractionally much larger, $45 \%$. Thus the resonance effects may be quite dramatic in state-selected quantities.

When we examine the state-to-state results, we see that many observables show non-monotonic behaviour in the vicinity of a resonance. Table 1 provides examples of some state-selected aspects of the results for the case with a 400 $\mathrm{cm}^{-1}$ gap. Table 1 shows factors of 4-5 changes in selected inelastic (i.e., non-reactive) probabilities of vibrational and rotational excitation. Looking at individual final states of $\mathrm{B}_{2}$, we see that there is a big resonance effect on the probability of producing $\mathrm{AB}$ in the $v^{\prime}=1, j^{\prime}=0$ state $(0.02$ increases to 0.10$)$ and a big enhancement for $v^{\prime}=0, j^{\prime}=2\left(4 \times 10^{-4}\right.$ increases to $1.8 \times 10^{-2}$ ), but only about a $10 \%$ effect for $v^{\prime}=0, j^{\prime}=8$. For reactive scattering, Table 1 shows a large effect for the probability of producing vibrationally excited $\mathrm{AB}$.

So far we have analysed the effect of coupling to the funnel in terms of the resonances that are exhibited when this coupling is turned on. Off resonance, the effect of coupling is typically small, $2-20 \%$. There are, however, a few exceptions, e.g., the rotational excitation process $\mathrm{A}+\mathrm{B}_{2}(v=j=0) \rightarrow \mathrm{A}$ $+\mathrm{B}_{2}\left(v^{\prime}=0, j^{\prime}=2\right)$. For the case with a $400 \mathrm{~cm}^{-1}$ gap, coupling to the funnel increases this probability by only $10 \%$ off resonance at low energy, but by a factor of 3 at some energies above the resonance. For example, this probability is $8 \times 10^{-4}$ at $0.97 \mathrm{eV}$ in the adiabatic case but increases to $2.2 \times 10^{-3}$ when coupling is turned on.

\section{Summary}

We have reported quantum mechanical calculations on a three-body reactive system that indicate the possibility of a new phenomenon, namely metastable states associated with a funnel located over a saddle point. Converged quantum dynamics calculations of energy-dependent reaction probabilities for a series of model atom-diatom reactions reveal structure associated with collisional resonances in which the three-body system is trapped in the funnel for times on the order of 25 fs. Resonances decrease the microcanonicalensemble reaction probability by $15-20 \%$, and the effect may be much larger for state-selected results, e.g., $45 \%$ for the reaction probability of vibrationally excited molecules. Offresonant effects of coupling to the funnel tend to be smaller, although off-resonant effects as large as a factor of 3 were observed in state-to-state non-reactive transition probabilities.

The authors are grateful to Greg Tawa for earlier contributions to the electronically non-adiabatic reactive scattering project. This work was supported in part by the National Science Foundation under grant no. CHE94-23927.

\begin{tabular}{|c|c|c|c|}
\hline \multirow[b]{2}{*}{ quantity } & \multicolumn{2}{|c|}{ typical value } & \multirow[b]{2}{*}{ resonance effect } \\
\hline & background & on resonance & \\
\hline inelastic vibrational excitation probability ${ }^{a, b}$ & 0.03 & 0.1 & factor of ca. 4 \\
\hline inelastic $v=j=0 \rightarrow v^{\prime}=0, j^{\prime}=2$ probability ${ }^{a}$ & 0.004 & 0.02 & factor of $c a .5$ \\
\hline total reaction probability ${ }^{c}$ & 0.67 & 0.59 & $-13 \%$ \\
\hline probability of $\mathrm{AB}\left(v^{\prime}=1\right) /$ prob. of $\mathrm{AB}\left(v^{\prime}=0\right)$ & 0.5 & 1.0 & factor of 2 \\
\hline mean $j^{\prime}$ of $\mathrm{AB}$ & 5 & $5 \frac{1}{2}$ & $10 \%$ \\
\hline
\end{tabular}

Table 1 Examples of the resonance effect on some state-stelected quantities (case $\mathrm{V}$, initial state has $v=j=0$ )

${ }^{a}$ We use 'inelastic' in the sense of non-reactive. ${ }^{b}$ Summed over $j^{\prime}=0,2,4$ for $v^{\prime}=1 .{ }^{c}$ Not state-selected. 


\section{Appendix}

\section{Functional forms of potential energy surfaces}

We consider the system $\mathrm{A}+\mathrm{BB}^{\prime}$. We begin with a set of Hamiltonian matrix elements that correspond to the type $\mathrm{B}^{\prime}$ crossing of valence bond configurations and that, when diagonalized, yield a result very similar to the $\mathrm{Cl}+\mathrm{H}_{2} \mathrm{G} 3$ surface as the lowest eigenvalue, which will be called $E_{1}^{*}$. We begin with a diabatic representation based on the London equation: ${ }^{39}$

$$
\begin{aligned}
& H_{11}=D_{\mathrm{BB}^{\prime}}+V_{\mathrm{BB}^{\prime}}^{S}+\frac{1}{4}\left(V_{\mathrm{AB}}^{S}+V_{\mathrm{AB}^{\prime}}^{S}\right)+\frac{3}{4}\left(V_{\mathrm{AB}^{T}}^{T}+V_{\mathrm{AB}^{\prime}}^{T}\right) \\
& H_{22}=D_{\mathrm{BB}^{\prime}}+\frac{3}{4}\left(V_{\mathrm{AB}^{S}}^{S}+V_{\mathrm{AB}^{\prime}}^{S}\right)+V_{\mathrm{BB}^{\prime}}^{T}+\frac{1}{4}\left(V_{\mathrm{AB}^{T}}^{T}+V_{\mathrm{AB}^{\prime}}^{T}\right)
\end{aligned}
$$

and

$$
H_{12} \equiv H_{21}=\frac{\sqrt{ } 3}{4}\left(V_{\mathrm{AB}}^{S}-V_{\mathrm{AB}^{\prime}}^{S}-V_{\mathrm{AB}^{\prime}}^{T}-V_{\mathrm{AB}}^{T}\right)
$$

where $D_{\mathrm{BB}^{\prime}}=4.747 \mathrm{eV}$, and $V_{\mathrm{BB}^{\prime}}^{S}, V_{\mathrm{BB}^{\prime}}^{T}, V_{\mathrm{AB}}^{S}$, and $V_{\mathrm{AB}}^{T}$ are taken from ref. 22. The diabatic coupling potential is then modified to make symmetry with respect to exchange of B and $\mathbf{B}^{\prime}$ manifest; in particular we found that we could do this without making a qualitatively undesirable change in the nature of the diabatic representation by replacing $H_{12}$ by

$$
H_{12}^{*}=\left\{\frac{\left(H_{12}\right)^{4}}{\left[a \exp \left(-2 \beta_{\mathrm{H}_{2}} \mathrm{R}_{\mathrm{H}_{2}}\right)\right]+\left(H_{12}\right)^{2}}\right\}
$$

where $a=333.9 \mathrm{meV}, \beta_{\mathrm{H}_{2}}=1.9413 \AA^{-1}$ and $R_{\mathrm{H}_{2}}$ is the $\mathrm{H}-\mathrm{H}$ distance. This Hamiltonian matrix is diagonalized numerically to yield the eigenvalues $E_{1}^{*}$ and $E_{2}^{*}$

$\left[\begin{array}{rr}\cos \gamma & \sin \gamma \\ -\sin \gamma & \cos \gamma\end{array}\right]\left[\begin{array}{ll}H_{11} & H_{12}^{*} \\ H_{12}^{*} & H_{22}\end{array}\right]\left[\begin{array}{cc}\cos \gamma & -\sin \gamma \\ \sin \gamma & \cos \gamma\end{array}\right]=\left[\begin{array}{cc}E_{1}^{*} & 0 \\ 0 & E_{2}^{*}\end{array}\right]$

We then calculate a new value, $E_{2}^{* *}$, for the upper adiabatic surface which gives the desired separation $\Delta E^{\ddagger}$ at the saddle point.

$$
E_{2}^{* *}=E_{2}^{*}-\left(1-\sin ^{4} \chi\right) z^{*}\left(E_{2}^{*}-E_{1}^{*}\right) z \exp (1-z)[\exp (-\Gamma)]
$$

where $\chi$ is the angle between the $\mathrm{B}-\mathrm{B}^{\prime}$ axis and a vector from A to the centre of mass of $\mathrm{BB}^{\prime}$, and

$$
\begin{gathered}
\Gamma=\left(\frac{r_{\mathrm{AB}}-r_{\mathrm{AB}}^{\ddagger}+r_{\mathrm{AB}^{\prime}}-r_{\mathrm{AB}^{\prime}}^{\ddagger}}{r_{1,0}}\right)^{4}+\left(\frac{r_{\mathrm{BB}^{\prime}}-r_{\mathrm{BB}}^{\ddagger}}{r_{2,0}}\right)^{4} \\
z=\frac{E_{2}^{*}-E_{1}^{*}}{\left(E_{2}^{*}-E_{1}^{*}\right)^{\ddagger}} \\
z^{*}=\frac{\left(E_{2}^{*}-E_{1}^{*}\right)^{\ddagger}-\Delta E^{\ddagger}}{\left(E_{2}^{*}-E_{1}^{*}\right)^{\ddagger}} \\
\left(E_{2}^{*}-E_{1}^{*}\right)^{\ddagger}=5.9184 \mathrm{eV}
\end{gathered}
$$

We obtained five surface sets by successively equating $\Delta E^{\ddagger}$ to (I) 1.24, (II) 12.40, (III) 24.80, (IV) 37.20, and (V) $49.59 \mathrm{meV}$. We then back transform to obtain a new Hamiltonian matrix

$$
\begin{aligned}
{\left[\begin{array}{rr}
\cos \gamma & -\sin \gamma \\
\sin \gamma & \cos \gamma
\end{array}\right]\left[\begin{array}{cc}
E_{1}^{*} & 0 \\
0 & E_{2}^{* *}
\end{array}\right]\left[\begin{array}{rr}
\cos \gamma & \sin \gamma \\
-\sin \gamma & \cos \gamma
\end{array}\right] } \\
=\left[\begin{array}{ll}
H_{11}^{\prime} & H_{12}^{\prime} \\
H_{12}^{\prime} & H_{22}^{\prime}
\end{array}\right]
\end{aligned}
$$

Finally, we introduce a 'fading' factor, $\lambda$,

$$
\lambda=\frac{1}{2}\left\{1-\tanh \left[\beta_{\mathrm{BB}^{\prime}}\left(r_{\mathrm{BB}^{\prime}}-r_{\mathrm{BB}^{\prime}}^{0}\right)\right]\right\}
$$

and transform to the final form of the diabatic Hamiltonian matrix

$$
\left[\begin{array}{ll}
H_{11}^{\mathrm{d}} & H_{21}^{\mathrm{d}} \\
H_{12}^{\mathrm{d}} & H_{22}^{\mathrm{d}}
\end{array}\right]=\left[\begin{array}{rr}
x & -y \\
y & x
\end{array}\right]\left[\begin{array}{ll}
H_{11}^{\prime} & H_{12}^{\prime} \\
H_{12}^{\prime} & H_{22}^{\prime}
\end{array}\right]\left[\begin{array}{rr}
x & y \\
-y & x
\end{array}\right]
$$

where

$$
\begin{gathered}
x=\left[\left(H_{11}^{\prime}-H_{22}^{\prime}\right)^{2}+4(1+\lambda) H_{12}^{\prime 2}\right] \\
\pm \frac{\left(H_{11}^{\prime}-H_{22}^{\prime}\right)+4\left(1-\lambda^{2}\right) H_{12}^{\prime 2}}{8 H_{12}^{\prime 2}+2\left(H_{11}^{\prime}-H_{22}^{\prime}\right)} \\
y= \pm \sqrt{1-x^{2}}
\end{gathered}
$$

The constant $\beta_{\mathrm{BB}^{\prime}}$ and $r_{\mathrm{BB}^{\prime}}^{0}$ were set equal to $1.9413 \AA^{-1}$ and $2.8046 \AA$, respectively.

We note for completeness that in ref. $48, H_{i j}^{\mathrm{d}}$ is called $V_{i j}$, the lower adiabatic surface $V_{1}$ is called $\bar{V}_{11}$, and the upper adiabatic surface $V_{2}$ is called $\bar{V}_{22}$. We note that our procedure does not require evaluating the non-adiabatic coupling (i.e., the coupling in the adiabatic representation) at any point in the calculation.

\section{References}

1 F. London, Probleme der Modernen Physik: Sommerfeld Festschrift, S. Herzel, Leipzig, 1928, p. 104; see also, W. Heitler and F. London, Z. Phys., 1927, 44, 455.

2 M. Born and J. R. Oppenheimer, Ann. Phys., 1927, 84, 457; M Born, Nachr. Akad. Wiss. Göttingen, 1951, 1; C. J. Ballhausen and A. E. Hansen, Annu. Rev. Phys. Chem., 1972, 23, 15; C. A. Mead, in Mathematical Frontiers in Computational Chemical Physics, ed. D. G. Truhlar, Springer-Verlag, New York, 1988, p. 8.

3 E. Teller, J. Phys. Chem., 1937, 41, 109

4 H. E. Zimmerman, J. Am. Chem. Soc., 1966, 88, 1566; H. E. Zimmerman, and R. E. Factor, J. Am. Chem. Soc., 1980, 102, 3538.

5 J. Michl, J. Mol. Photochem., 1972, 4, 243.

6 J. Michl, and V. Bonačić-Koutecký, Electronic Aspects of Organic Photochemistry, John Wiley \& Sons, New York, 1990.

7 W. Domcke, A. L. Sobolewski and C. Woywood, Chem. Phys Lett., 1993, 203, 220; I. J. Palmer, I. N. Ragazos, F. Bernardi, M. Olivucci and M. A. Robb, J. Am. Chem. Soc., 1993, 115, 673; M Olivucci, I. N. Ragazos, F. Bernardi and M. A. Robb, J. Am. Chem. Soc., 1993, 115, 3710; F. Bernardi, M. Olivucci and M. Robb, Isr. J. Chem., 1993, 33, 265.

8 H. A. Jahn and E. Teller, Proc. R. Soc. London, Ser. A, 1937, 161, 220; C. A. Mead, J. Chem. Phys., 1979, 70, 2276; 1980, 72, 6817.

9 L. Salem, Electrons in Chemical Reactions: First Principles, John Wiley \& Sons, New York, 1982

10 F. London, Z. Elektrochem., 1929, 35, 552.

11 H. Eyring and M. Polanyi, Z. Phys. Chem., 1929, 35, 552; S. Sato, Bull. Chem. Soc. Jn., 1955, 28, 450; J. Chem. Phys., 1955, 23, 592; J. K. Cashion and D. R. Herschbach, J. Chem. Phys., 1964, 40, 2358; C. A. Parr and D. G. Truhlar, J. Phys. Chem., 1971, 75, 1844; D. G. Truhlar and R. E. Wyatt, Adv. Chem. Phys., 1977, 36, 141.

12 I. Yasumori, Bull. Chem. Soc. Jn, 1959, 32, 1103; 1959, 32, 1110; R. N. Porter, R. M. Stevens and M. Karplus, J. Chem. Phys., 1968, 49, 5163.

13 S. S. Shaik, J. Am. Chem. Soc., 1981, 103, 3692; S. Shaik and P. C. Hiberty in Theoretical Models of Chemical Bonding, Z. B. Maksic, Springer-Verlag, Heidelberg, 1991, vol. 4, p. 269; S. Shaik, A. Ioffe, A. C. Reddy and A. Pross, J. Am. Chem. Soc., 1994, 116, 262; S. Shaik and P. C. Hiberty, Adv. Quantum Chem., 1995, 26, 99.

14 S. Shaik and A. C. Reddy, J. Chem. Soc., Faraday Trans., 1994 90, 1631; M. Olivucci, J. Chem. Soc., Faraday Trans., 1994, 90 1670; D. G. Truhlar, J. Chem. Soc., Faraday Trans., 1994, 90 1670 .

15 J. Callaway, Quantum Theory of the Solid State, Academic Press, New York, 1974.

16 R. A. Marcus, in Tunneling in Biological Systems, ed. B. Chance, D. DeVault, H. Fraunfelder, R. A. Marcus, J. R. Schreiffer and N. 
Sutin, Academic Press, New York, 1979, p. 109; M. Bixon and J. Jortner, Faraday Discuss. Chem. Soc., 1982, 74, 17.

17 M. D. Pearson, P. W. Kash and L. J. Butler, J. Chem. Phys., 1992, 97, 355; G. C. G. Waschewsky, P. W. Kash, T. L. Myers, D. C. Kitchen and L. J. Butler, J. Chem. Soc., Faraday Trans., 1994, 90, 1581.

18 R. B. Woodward and R. Hoffmann, The Conservation of Orbital Symmetry, Verlag Chemie, Weinheim, 1970.

19 T. F. O’Malley, Adv. At. Mol. Phys., 1971, 7, 223; B. C. Garrett and D. G. Truhlar, Theor. Chem. (NY), 1981, 6A, 215; H. Köppel, W. Domcke and L. S. Cederbaum, Adv. Chem. Phys., 1984, 56, 59; V. Sidis, Adv. Chem. Phys., 1992, 82, 73; T. Pacher, L. S. Cederbaum and H. Köppel, Adv. Chem. Phys., 1993, 84, 293.

20 A. Sevin, C. Giessner-Prettre, P. C. Hiberty and E. Noizet. J. Am. Chem. Soc., 1991, 95, 8580.

21 H. E. Zimmerman, Acc. Chem. Res., 1972, 5, 393; D. G. Truhlar, J. Chem. Soc., Faraday Trans., 1994, 90, 1614

22 T. C. Allison, G. C. Lynch, D. G. Truhlar and M. S. Gordon, J. Chem. Phys., 1996, 100, 13575.

23 A. G. Evans and M. G. Evans, Trans. Faraday Soc., 1935, 31, 1400; J. J. Ewing, R. Milstein and R. S. Berry, J. Chem. Phys., 1971, 54, 1752.

24 I. J. Palmer, I. N. Ragazos, F. Bernardi, M. Olivucci and M. A. Robb, J. Am. Chem. Soc., 1994, 116, 2121.

25 B. C. Garrett and D. G. Truhlar, J. Chem. Phys., 1985, 82, 4543 ; 1986, 84, 7057 (E); A. G. Ioannov, R. D. Amos and N. C. Handy, Chem. Phys. Lett., 1996, 251, 52.

26 (a) L. Landau, Phys. Z. Sowjetunion, 1932, 2, 46; (b) C. Zener, Phys. Rev., 1932, 137, 696; (c) E. C. G. Stückelberg, Helv. Phys. Acta, 1932, 5, 369.

27 E. E. Nikitin and S. Y. Umanskii, Theory of Slow Atomic Collisions, Springer-Verlag, Berlin, 1984.

28 H. Nakamura, Int. Rev. Phys. Chem., 1991, 10, 123; H. Nakamura, J. Chem. Phys., 1992, 97, 256; C. Zhu and H. Nakamura, J. hem. Phys., 1994, 101, 4855; H. Nakamura, Comments At. Mol. Phys., 1996, 32, 249; H. Nakamura, in Dynamics of Molecules and Chemical Reactions, ed. R. E. Wyatt and J. E. H. Zhang, Marcel Dekker, New York, 1996, p. 473.

29 S. Shin and J. C. Light, J. Chem. Phys., 1994, 101, 2836; J. Qi and J. M. Bowman, J. Chem. Phys, 1996, 104, 7545.

30 A. Bjerre and E. E. Nikitin, Chem. Phys. Lett., 1967, 1, 179, J. C. Tully and R. K. Preston, J. Chem. Phys., 1971, 55, 562.

31 D. G. Truhlar and J. T. Muckerman, in Atom-Molecule Collision Theory, ed. R. B. Bernstein, Plenum, New York, 1979, p. 505; D. G. Truhlar and D. A. Dixon, in Atom-Molecule Collision Theory, ed. R. B. Barnstein, Plenum, New York, 1979, p. 595.

32 Resonances, ed. D. G. Truhlar, American Chemical Society, Washington, DC, 1984

33 M. M. Kreevoy and D. G. Truhlar, in Investigation of Rates and Mechanisms of Reactions, ed. C. F. Bernasconi, John Wiley, New York, 1986, pt. I, p. 13; V. A. Benderskii, D. E. Makarov and C. A. Wight, Adv. Chem. Phys., 1994, 88, 1.

34 M. Gilibert and M. Baer, J. Phys. Chem., 1994, 98, 12822; 1995, 99, 15748.

35 S. L. Mielke, G. J. Tawa, D. G. Truhlar and D. W. Schwenke, Chem. Phys. Lett., 1995, 234, 57.
36 S. L. Mielke, D. G. Truhlar and D. W. Schwenke, J. Phys. Chem., 1995, 99, 16210 .

37 G. C. Schatz, J. Chem. Phys., 1995, 99, 7522.

38 A. J. C. Varandas and H. G. Yu, Chem. Phys. Lett., 1996, 259, 336.

39 B. C. Garrett and D. G. Truhlar, J. Chem. Phys., 1985, 82, 4543.

40 D. W. Schwenke, S. C. Tucker, R. Steckler, F. B. Brown, G. C. Lynch, D. G. Truhlar and B. C. Garrett, J. Chem. Phys., 1989, 90, 3110.

41 R. G. Newton, Scattering Theory of Particles and Waves, McGraw-Hill, New York, 1966, sect. 11.3.

42 D. W. Schwenke, K. Haug, D. G. Truhlar, Y. Sun, J. Z. H. Zhang, and D. J. Kouri, J. Phys. Chem., 1987, 91, 6080.

43 D. W. Schwenke, K. Haug, M. Zhao, D. G. Truhlar, Y. Sun, J. Z. H. Zhang and D. J. Kouri, J. Phys. Chem., 1988, 92, 3202.

44 L. Schlessinger, Phys. Rev., 1968, 187, 1411

45 Y. Sun, D. J. Kouri, D. G. Truhlar and D. W. Schwenke, Phys. Rev. A, 1990, 41, 4857.

46 Y. Sun, D. J. Kouri, and D. G. Truhlar, Nucl. Phys. A., 1990, 508, 41c.

47 D. W. Schwenke, S. L. Mielke and D. G. Truhlar, Theor. Chim. Acta, 1991, 79, 241.

48 G. J. Tawa, S. L. Mielke, D. G. Truhlar and D. W. Schwenke, in Advances in Molecular Vibrations and Collision Dynamics, vol. 2B, ed. J. M. Bowman, JAI Press, Greenwich, CT, 1994, pp. 45-116.

49 G. J. Tawa, S. L. Mielke, D. G. Truhlar and D. W. Schwenke, J. Chem. Phys., 1994, 100, 5751.

50 W. H. Miller, J. Chem. Phys, 1975, 62, 1899; D. G. Truhlar, A. D. Isaacson and B. C. Garrett, in Theory of Chemical Reaction Dynamics, ed. M. Baer, CRC Press, Boca Raton, FL, 1985, vol. 4, p. 127; J. M. Bowman, A. F. Wagner, in The Theory of Chemical Reaction Dynamics, ed. D. C. Clary, Reidel, Dordrecht, 1986, p. 47; S. L. Mielke, G. C. Lynch, D. G. Truhlar and D. W. Schwenke, Chem. Phys. Lett., 1993, 216, 441.

51 D. C. Chatfield, R. S. Friedman, D. G. Truhlar, B. C. Garrett and D. W. Schwenke, J. Am. Chem. Soc., 1991, 113, 486.

52 J. R. Taylor, Scattering Theory, John Wiley, New York, 1972, p. 242; A. Bohm, Quantum Mechanics: Foundations and Applications, 3rd edn., Springer-Verlag, New York, 1993, pp. 493-497.

53 R. G. Newton, Scattering Theory of Waves and Particles, 2nd edn, Springer-Verlag, New York, 1982, p. 621, M. Zhao, M. Mladenovic, D. G. Truhlar, D. W. Schenke, O. Sharafeddin, Y. Sun and D. J. Kouri, J. Chem. Phys., 1989, 91, 5302.

54 D. C. Chatfield, R. S. Friedman, S. L. Mielke, G. C. Lynch, T. C. Allison, D. G. Truhlar and D. W. Schwenke, in Dynamics of Molecules and Chemical Reactions, ed. R. E. Wyatt and J. Z. H. Zhang, Marcel Dekker, New York, 1996, p. 323.

55 T. C. Allison, S. L. Mielke, D. W. Schwenke, G. C. Lynch, M. S. Gordon and D. G. Truhlar, in Gas-Phase Chemical Reactions: Experiments and Models 100 Years after Max Bodenstein, ed. J. Wolfrum, H-R. Volpp, R. Rannacher and J. Warnatz, Springer, Heidelberg, 1996, pp. 111-124.

56 S. L. Mielke, T. C. Allison, D. G. Truhlar and D. W. Schwenke, J. Phys. Chem., 1996, 100, 13588.

Paper 6/06254G; Received 10th September, 1996 